\title{
Dynamic Simulation on Vehicle during Spiral Rolling
}

\author{
Xu Zhang ${ }^{1, a}$, Da Song ${ }^{1, b}$, Wenxiu Shang ${ }^{1, c}$, Lixun Zhang ${ }^{1, d}$, \\ Kunlun $\mathrm{Wu}^{1, \mathrm{e}}$, Yiwen Zhang ${ }^{1, \mathrm{f}}$, Qihe Zhang $^{1, \mathrm{~g}}$ \\ ${ }^{1}$ Harbin Engineering University, Harbin 150001, P. R. China \\ azhangxu@hrbeu.edu.cn, 'songda@hrbeu.edu.cn, c'shangwenxiu@hrbeu.edu.cn, 'zhanglixun@hrbeu.edu.cn, \\ ewukunlun@hrbeu.edu.cn, zhangyiwen@hrbeu.edu.cn and ${ }^{9}$ zhangqihe@hrbeu.edu.cn
}

Keywords: spiral rolling; vehicle; numerical simulation; dynamic explicit finite element analysis

Abstract. Dynamic explicit finite element analysis is practical and significant to study vehicle spiral rolling. By means of HyperMesh and LsDyna, numerical simulation is achieved with deformation, posture and acceleration obtained. During spiral rolling process, vehicle roof comes much close to occupant head step by step, so vehicle roof should be intensified and optimized moderately. Initial impacting velocity is set as $80 \mathrm{~km} / \mathrm{h}$, dummy does not leave the seat until the rolling ends with safety belt adopted. While, without safety belt, dummy detaches or contacts vehicle for several times, head and neck have relatively large deflection and bending. Without the protection of the safety belt, the injury of head, neck and chest during spiral rolling goes beyond the regulation requirements for safety, serious casualties will occur consequentially.

\section{Introduction}

Dynamic explicit finite element analysis is practical and significant to study vehicle spiral rolling, not only being able to evaluate accurately occupant injury and integrity of occupant compartment after a rolling accident, but also to predict the crash worthiness of vehicle body [1]. Vehicle spiral rolling test has been widely promoted and applied in Europe and United States. Therein, the tested vehicle moves to a fixed slope at a specified initial velocity [2]. Wheels on one side of the vehicle go along the ground and wheels on the other side go along the slope upward. It is easy for height difference between wheels on two sides to bring on the occurrences of spiral rolling accident [3].

\section{The model establishment for dynamic simulation}

Vehicle spiral rolling simulation is carried out based on the following assumptions: (1) In the whole process, except tire contact with the slope, the vehicle doesn't contact with other objects such as other vehicles, fences and trees. (2) Vehicle steering system is ignored, driver has no emergency response. By means of dynamic explicit finite element method, numerical simulation is achieved on vehicle during spiral rolling, with HyperMesh and LsDyna applied.

The spiral rolling of vehicle. The simulation duration of vehicle spiral rolling is set as $1.5 \mathrm{~s}$, vehicle posture during spiral rolling is shown in Fig.1. The vehicle movement posture obtained from this simulation is consistent fairly, compared with that from real vehicle rolling test, which indicates that the finite element model and the relevant settings are correct and reliable for further research.

The deformation analysis of vehicle roof. Fig. 2 shows the deformation of vehicle roof during spiral rolling. Because centroid is near the front of the vehicle, vehicle rear goes upward while front sinks during spiral rolling, which conduces front roof deformed greatly. It can be seen that rolling begining from left side generally leads to a more serious deformation at the right side of the roof.

Roof intrusion is an important index for evaluating living space during and after spiral rolling [4]. In order to observe roof deformation well, the intrusion value of vehicle roof during spiral rolling is given in Fig.3. Roof intrusion value gradually increases with the rolling process, and the maximum intrusion value reaches up to $118.4 \mathrm{~mm}$, which indicates that the vehicle roof comes much close to occupant head step by step, so vehicle roof should be intensified and optimized moderately. 

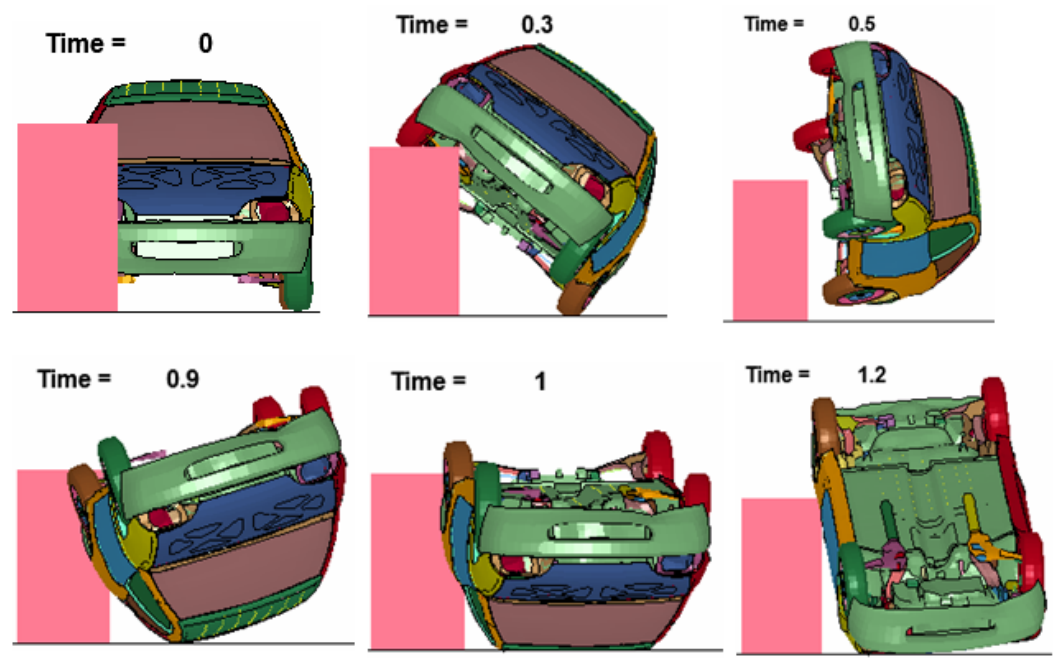

Fig.1 Vehicle posture during spiral rolling
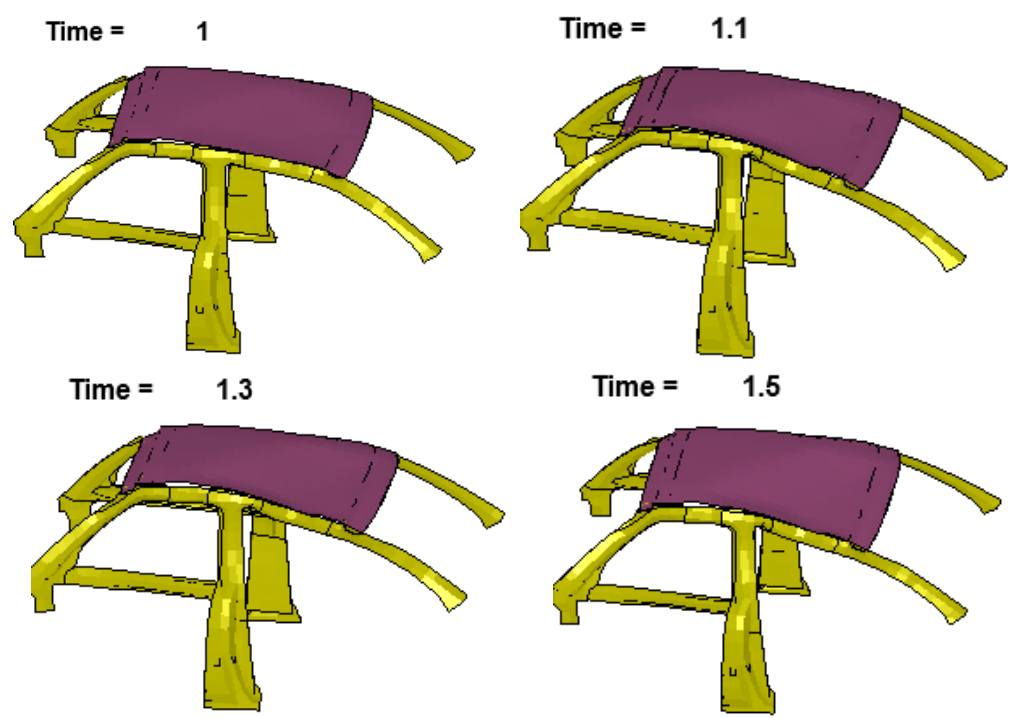

Fig.2 Deformation of vehicle roof during spiral rolling

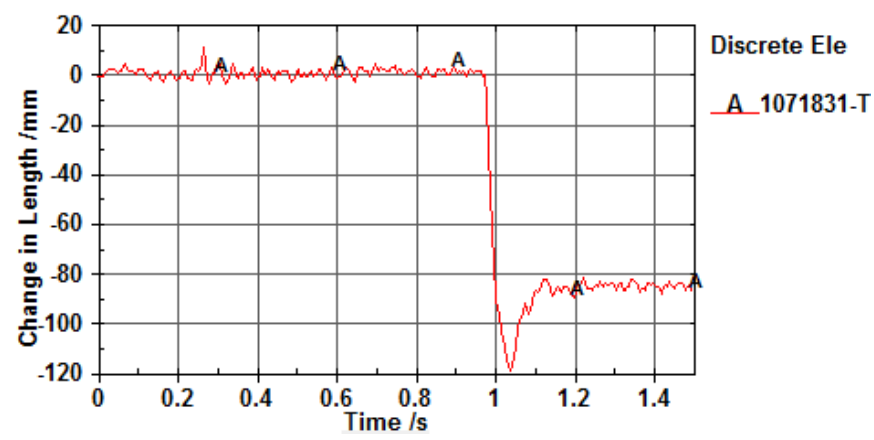

Fig.3 The intrusion value of vehicle roof during spiral rolling

\section{Dummy analysis during spiral rolling}

In order to adapt to the most severe testing conditions, initial impacting velocity is set as $80 \mathrm{~km} / \mathrm{h}$, allowable maximum value for spiral rolling test, and dummy locates in the vehicle. Dummy posture in the vehicle during spiral rolling is indicated in Fig.4. With a safety belt adopted, inertia effect makes dummy move to the left side of the rolling vehicle when time $t=0.2 \mathrm{~s}$. $\mathrm{T}=0.5 \mathrm{~s}$, tire runs away from the slope, dummy head and neck have almost not any apparent deflection, two legs have small 
swing outward, arms have some contact with seat. $\mathrm{T}=0.8 \mathrm{~s}$, dummy head starts to deviate from the seat due to side shift. $T=1.2 \mathrm{~s}$, hand swing amplitude increases further, at the same time, left leg begins to swing to the vehicle roof, dummy does not leave the seat until the rolling ends. However without the safety belt, when time $t=0.5 \mathrm{~s}$, inertia makes the dummy roll to the opposite side of the vehicle, then the dummy moves along the rolling way. Dummy head contacts with vehicle roof when $t=1 \mathrm{~s}$, leading to large deflection of the head and neck bending, and the dummy is thrown off the seat a little. $T=1.3 \mathrm{~s}$, dummy is completely thrown off the seat. During the rolling, dummy detaches or contacts vehicle for several times, head and neck have relatively large deflection and bending.

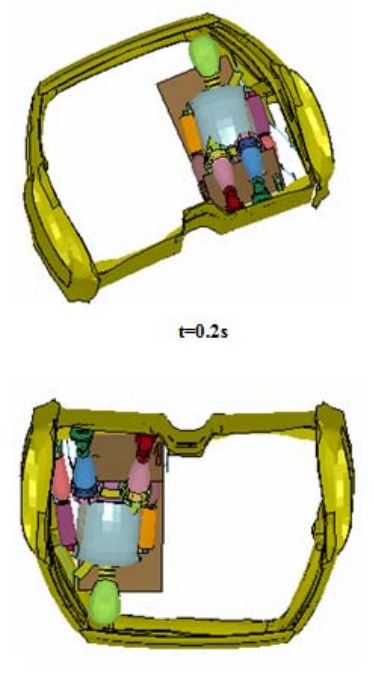

$t=0.8 \mathrm{~s}$

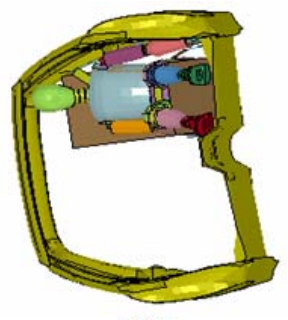

$\mathrm{t}=0.5 \mathrm{~s}$

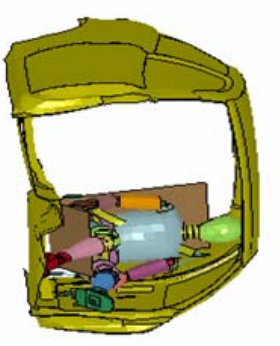

$t=1.25$

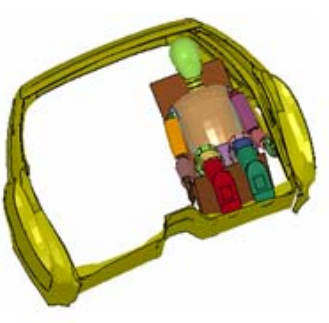

$t=0.2 s$

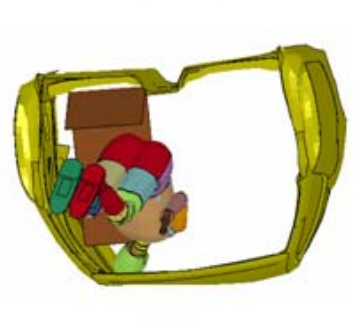

$t=1 s$

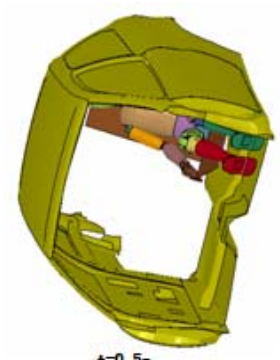

$t=0.5 \mathrm{~s}$

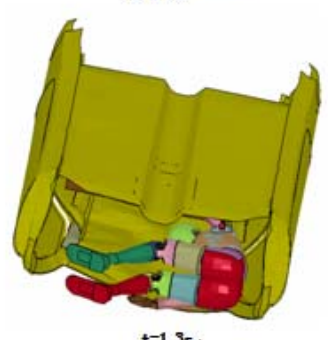

$t=1.3 s$.

(a)

(b)

Fig.4 Dummy posture in the vehicle during spiral rolling
(a) With safety belt
(b) Without safety belt

The acceleration of dummy head during spiral rolling is shown in Fig.5. Under the protection of three point type safety belt, dummy head bears maximum effective acceleration 82g, which exceeds the requirements of acceleration regulation a little. And maximum value of head injury criterion Hic $_{36}$ is 404.3. Although the $\mathrm{Hic}_{36}$ value is far lower than regulation requirements, the dummy still subjects to serious damage just because of higher head acceleration. Without the protection of the safety belt, maximum acceleration of dummy head is up to $297.3 \mathrm{~g}$, coresponding $\mathrm{Hic}_{36}$ reaches up to $3054 \mathrm{~g}$, which indicates that the human body damage is fatal without safety belt. And at the same time, by analyzing the head acceleration and $\mathrm{Hic}_{36}$ values when the same dummy locates on the different side of the rolling vehicle, the damage grade in the far side of the rolling vehicle is significantly much higher than that in the near side. Hereafter, the dummy neck, neck torque, chest compression degree are also analyzed. Without the protection of the safety belt, the injury of head, neck and chest during spiral rolling goes beyond the regulation requirements for safety, serious casualties will occur consequentially. 


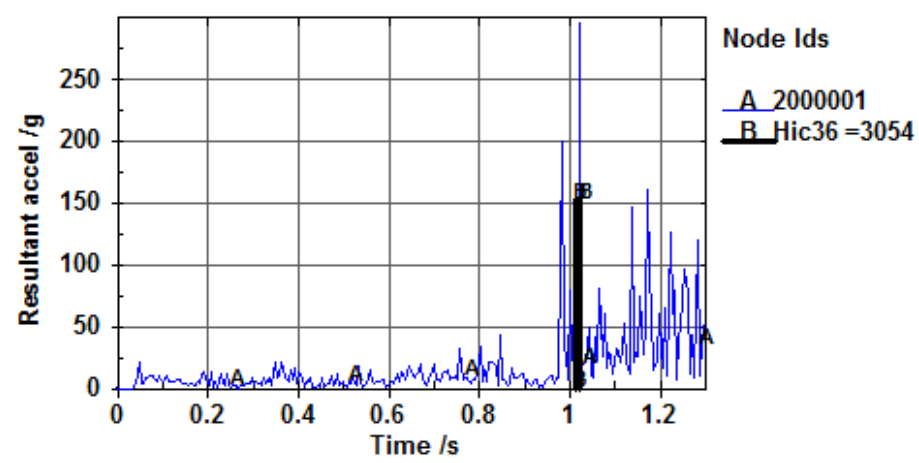

Fig.5 The acceleration of dummy head during spiral rolling

\section{Conclusions}

By means of dynamic explicit finite element method, numerical simulation is achieved on vehicle during spiral rolling. Some conclusions are drawn as follows. During spiral rolling process, vehicle roof comes much close to occupant head step by step, so vehicle roof should be intensified and optimized moderately. Initial impacting velocity is set as $80 \mathrm{~km} / \mathrm{h}$, dummy does not leave the seat until the rolling ends with safety belt adopted. While, without safety belt, dummy detaches or contacts vehicle for several times, head and neck have relatively large deflection and bending. Without the protection of the safety belt, the injury of head, neck and chest during spiral rolling goes beyond the regulation requirements for safety, serious casualties will occur consequentially.

\section{Acknowledgements}

We gratefully acknowledge the help of Dr. Xiaodong Xing. This thesis is supported by NSFC, China (51179040).

\section{References}

[1] Hu Zhiyuan, Zeng Biqiang, Xie Shugang. Based on LS-DYNA and HyperWorks Automotive Safety Simulation Analysis [M]. Master Thesis. Tsinghua University Press. (2011). (In Chinese)

[2] Tao Yi. Study on Characteristics of New Vehicle Collision Energy Absorber [D]. Shanghai Jiaotong University.( 2005). (In Chinese)

[3] Yu Xuebing, Mao Juanjuan, Liu Liping. Simulation Analysis of Semi Rigid Three Wave Barrier and Passenger Vehicle [J]. Automotive Technolog. 2009(3): 54-57. (In Chinese)

[4] Chuck A. Plaxico. Comparison of the impact performance of the G4(1W) and G4(2W) guardrail systems under CHRP Report 350 test 311 conditions. Transportation Research Record Journal of the Transportation Research Board. 2000, 1720:7-18 